\title{
Raman spectroscopic investigation of frozen and deparaffinized tissue sections of pediatric tumors: neuroblastoma and ganglioneuroma
}

\author{
Suneetha Devpura, ${ }^{a}$ Jagdish S. Thakur, ${ }^{a}$ Janet M. Poulik, ${ }^{b}$ Rajah Rabah, ${ }^{c}$ \\ Vaman M. Naik ${ }^{\mathbf{d}_{*}}$ and Ratna Naik ${ }^{\mathrm{a}}$
}

Raman spectroscopy is a nondestructive technique that can provide information at the molecular level about the biochemicals in tissues. We have investigated the cellular regions in neuroblastoma and ganglioneuroma using Raman spectroscopy and compared their spectral characteristics with those of the corresponding normal adrenal gland. Thin sections from both the frozen and the corresponding formalin-fixed paraffin-processed (FFPP) tissues were studied in conjunction with the pathological examination of the tissues. Investigation of the spectral data shows that the normal adrenal gland tissues have higher levels of carotenoids, lipids, and cholesterol compared with the neuroblastoma and ganglioneuroma frozen tissues. However, in comparison with the frozen tissues, the FFPP tissues show a significant alteration of several biochemicals, including the complete removal of carotenoids, lipids, and cholesterol in the adrenal tissues. A quantitative analysis using chemometric methods of principal component analysis and discriminant function analysis of the Raman spectral data obtained from the frozen tissues show a clear-cut classification among pathological groups with high sensitivity and specificity. We have validated the classification results of the FFPP tissues against a training set data obtained from the archived FFPP tissues of nine other patients. The validation process correctly identified and grouped the data with the training set of normal adrenal gland ( $>97 \%$ of the time) and neuroblastoma ( $100 \%$ of the time) tissues, whereas the validation was not so strong for ganglioneuroma. This study shows that Raman spectroscopy combined with chemometric methods can be successfully used to distinguish neuroblastoma and ganglioneuroma at cellular level in frozen tissue sections. This study also shows that formalin fixation and paraffinization/deparaffinization of tissues can alter their biochemical composition. Copyright $\odot 2012$ John Wiley \& Sons, Ltd.

Supporting information may be found in the online version of this article.

Keywords: Raman spectroscopy; neuroblastoma; ganglioneuroma; frozen tissue; deparaffinized tissue

\section{Introduction}

Neuroblastoma and ganglioneuroma are peripheral neuroblastic tumors derived from the immature sympathetic nervous system and the adrenal gland. Neuroblastoma, the third most common type of children cancer, is a highly malignant tumor that affects the sympathetic peripheral nervous system. It is frequently unamenable to surgical removal at diagnosis and requires chemotherapy followed by surgical debulking. Prognosis is dependent on the patient's age, morphological findings such as degree of ganglionic differentiation, stromal maturation, mitotic/karyorrhectic count, and biological molecular markers. ${ }^{[1]}$ Ganglioneuroma on the other hand is a benign neoplasm that is considered the end result of spontaneous maturation of neuroblastoma in a subset of patients. This is a unique phenomenon that is still incompletely understood. Ganglioneuromas are usually treated by surgical removal, no chemotherapy is needed, and the prognosis is excellent. Chemotherapy treatment in neuroblastoma also induces ganglionic maturation and stromal maturation, and often, neuroblastomas at the time of debulking surgery appear histologically similar to ganglioneuromas. ${ }^{[2]}$

Recently, Raman spectroscopy has been used in the investigation of pediatric tumor specimens. ${ }^{[3-6]}$ Rabah et al. compared Raman spectra of several pediatric tumors in biopsy or resection of fresh tissue specimens and showed that the normal adrenal gland tissues contain a high concentration of carotenoids compared with neuroblastoma, ganglioneuroma, and nerve sheath tumors. $^{[3]}$ The effect of cryopreserving the tissue specimens, by freezing to $-80^{\circ} \mathrm{C}$, shows no significant changes in the Raman spectra of the frozen tissues compared with the fresh ones. ${ }^{[4]}$ Wills et al. have successfully used Raman spectroscopy to identify Wilms' tumor and a variety of other related renal tumors using both fresh and frozen tissue specimens. ${ }^{[5]}$ Min et al. have studied neuroblastoma bulk specimens preserved in formaldehyde using 1064-nm excitation laser. ${ }^{[6]}$ They observed changes in the intensity of Raman bands $\sim 1440$ and $\sim 1303 \mathrm{~cm}^{-1}$ with their intensity

\footnotetext{
* Correspondence to: Vaman M. Naik, Department of Natural Sciences, University of Michigan-Dearborn, Dearborn, MI 48128, USA.

E-mail:vmnaik@umich.edu

a Department of Physics and Astronomy, Wayne State University, Detroit, M 48201, USA

b Department of Pathology, Children's Hospital of Michigan, Detroit, MI 48201, USA

c Department of Pathology, University of Michigan, Ann Arbor, MI 48109, USA

d Department of Natural Sciences, University of Michigan-Dearborn, Dearborn, MI 48128, USA
} 
ratio being larger than one in normal adrenal tissue, whereas it was comparable with or smaller than one in neuroblastomas. On the basis of this observation, they proposed that this ratio can be used to differentiate neuroblastomas from the normal adrenal tissues.

Raman spectroscopy has often been used to study the fresh tissues. Recent studies have shown that the spectra from fresh and frozen tissues show no significant differences, ${ }^{[4,5]}$ thus enhancing the utility of this technique to study the banked frozen tissues to better define characteristic profiles of different tumors. However, there is limited availability of the fresh and frozen banked tissue to allow studies with large number samples. Although several investigations have been carried out on frozen tissues, the abundant availability of archived formalin-fixed paraffin-preserved (FFPP) tissues may provide a venue for conducting a thorough investigation for diagnostic purpose. However, the preservation of tissue specimens by embedding them in paraffin wax is known to alter its biochemical composition. In a study using human placenta specimens, it has been shown that formalin fixation results in additional peaks and shifts in the amide bands resulting from changes in protein conformation and possible cross-links. ${ }^{[7]}$ Thus, for a reliable diagnosis of pathological state in deparaffinized tissues using Raman spectroscopy, it is important to identify the biochemical changes that occur in the archived FFPP tissues. Once the nature of the loss of biochemical information is known and understood, one can pursue further studies using FFPP tissues. Further, the pathological evaluations have inherently an element of subjectivity as the outcome of the evaluation is based on the experience and intuition of the pathologist. The information extracted from the pathological evaluations has a high impact in determining the treatment strategy. Hence, it is crucial to develop an objective optical technique such as Raman spectroscopy to diagnose the pathological state of a tissue with high reliability. The diagnostic ability of Raman spectroscopy for paraffin-embedded archived pediatric tumors/neoplasm has not yet been fully explored.

In the present study, we have used Raman spectroscopy to investigate frozen tissue sections of normal adrenal gland, neuroblastoma, and ganglioneuroma obtained from three different patients, and compared their Raman spectral features with the corresponding deparaffinized sections of FFPP tissues. The goal of this study is to understand the nature of biochemical changes that occur in FFPP tissues and to assess their feasibility for diagnosis of diseases using Raman spectroscopy. We have attempted to determine the Raman spectroscopic signatures of each type of tumor, particularly, from the cellular regions of well-identified pathology rather than collecting data from random sites in the bulk tissue specimens as was carried out in the earlier studies. ${ }^{[3-5]}$ The quantitative analysis of the Raman data using chemometric methods of principal component analysis (PCA) and discriminant function analysis (DFA) obtained from both the frozen and the FFPP tissues shows a clear-cut classification among pathological groups. We also discuss an external validation of our classification against a training data set obtained from the archived FFPP tissues of nine other patients.

\section{Materials and methods}

\section{Raman spectrometer}

Raman spectra were collected with a Renishaw inVia Raman microscope spectrometer using a 785-nm line from a diode laser.
A $50 \times$ objective was used with a microscope that focused the laser light into a line of $\sim 2 \times 20 \mu \mathrm{m}^{2}$ in area. The resolution of the spectrometer using 1200 grooves $/ \mathrm{mm}$ grating was $\sim 3.5 \mathrm{~cm}^{-1}$. The Raman instrument was calibrated using Si standard prior to each measurement session.

\section{Tissue preparation}

For the comparison study of frozen versus deparaffinized specimens using Raman spectroscopy, the tissues were obtained from three patients from the Children's Hospital of Michigan, Detroit, MI. Patient \#1 is a 3-year-old infant with adrenocortical tumor present in right adrenal gland. A normal adrenal gland specimen was obtained from this patient. The pathology examination verified the absence of any malignant cells in the tissue. Patient \#2 is an 18-month-old female infant with neuroblastoma. The specimen was obtained from the left adrenal gland. The tumor $(12 \mathrm{~cm}$ in dimension and weighed $0.510 \mathrm{~kg}$ ) was poor in stroma and was poorly differentiated. This neuroblastoma specimen has been identified as having a favorable histology, and pathological evaluation showed this specimen contains nests of tumor cells with centering neuropils. Patient \#3 is a 7-year-old child with ganglioneuroma. The specimen is a spinal mass having differentiating mature ganglions in a dominant Schwannian stroma environment. The tumor was $3 \times 2 \times 1.5 \mathrm{~cm}$ in size, and clusters of ganglions were observed throughout the specimen. First, the specimens from the three patients were thawed for about $20 \mathrm{~min}$, and then, the desired frozen sections were cut with a microtome using optimal cutting temperature solution. From each tissue specimen, a 5 - $\mu$ m-thick section was cut and stained with hematoxylin and eosin (H\&E) for pathological examination, and additional three adjacent $10-\mu \mathrm{m}$-thick parallel sections were cut for Raman measurements. The remaining tissue specimen, keeping the same orientation, was put in formalin for about $24 \mathrm{~h}$ and subsequently preserved in paraffin wax. From this FFPP tissue block, four additional parallel sections were cut, one for H\&E staining and the rest for Raman spectroscopic investigation. The paraffin was removed from the tissue sections using the standard deparaffinizing procedure described in the work of Devpura et al. ${ }^{[8]}$ Additionally, we have also studied archived FFPP tissues from nine other patients. The purpose of this study was to use this data as a training set and test the data from the deparaffinized tissues from the earlier part of the study. The details of the pathologies of the additional nine patients are given in Table 1. Tissue sections were cut from these samples for pathological examination and Raman spectroscopic investigation as described previously. No frozen specimens were available for these cases.

All the H\&E stained slides used in this study were examined by two experienced pathologists who marked the regions of interest on the slides, and the images were taken from each pathological condition of the tissues. We used the pathologically examined/marked H\&E slides of the tissue as a guide to identify the corresponding locations of appropriate pathology in the unstained tissue sections of frozen or deparaffinized tissues placed on stainless steel substrate to collect the Raman spectra. We assume that the pathological state of the tissue in the $10-\mu \mathrm{m}$-thick sections is the same as in the adjacent $5-\mu \mathrm{m}$ thick tissue section used by the pathologists. We have used three tissues from frozen and deparaffinized samples to collect the Raman data. 
Table 1. The pathologies and the number of Raman spectra obtained from formalin-fixed paraffin-processed tissues of nine patients

\begin{tabular}{|c|c|c|c|c|}
\hline Case number & Patient number & Pathology & Tissue source & Raman spectra \\
\hline 1 & P1 & Normal & Adrenal & 78 \\
\hline 2 & P2 & Normal & Adrenal & 11 \\
\hline 3 & P3 & Normal & Adrenal & 36 \\
\hline 4 & P3 & Ganglioneuroma & Adrenal & 8 \\
\hline 5 & P4 & Ganglioneuroma & Extradural spinal cord mass & 20 \\
\hline 6 & P5 & Ganglioneuroma & Abdominal mass & 40 \\
\hline 7 & P6 & Ganglioneuroma & Retroperitoneal mass & 71 \\
\hline 8 & P7 & Neuroblastoma & Retroperitoneal mass & 37 \\
\hline 9 & P8 & Neuroblastoma & Posterior mediastinal mass & 68 \\
\hline 10 & P9 & Neuroblastoma & Paraspinal mass & 12 \\
\hline
\end{tabular}

\section{Data collection}

For a comparison study of frozen versus deparaffinized specimens, a total of 558 Raman spectra were collected: 205 from normal adrenal gland (103 frozen and 102 deparaffinized), 201 were from neuroblastoma (101 frozen and 100 deparaffinized), and 196 were from ganglioneuroma (102 frozen and 94 deparaffinized).

In the second part of the study, the FFPP tissue specimens from nine more patients were investigated. The details of the pathologies of these patients and the number of spectra collected are given in Table 1. A total of 381 Raman spectra were collected from the tissue specimens. Each spectrum is an average of three accumulations with 20-s integration time. The power at the sample was $\sim 20 \mathrm{~mW}$. The Raman spectra were recorded in the range of $500-1900 \mathrm{~cm}^{-1}$. Each spectrum was recorded in $\sim 4 \mathrm{~min}$ on a fresh spot of the tissue.

\section{Data analysis}

Each Raman spectrum was visually examined, and any cosmic ray noise was removed. The fluorescence background present in the spectrum was removed using a customized MATLAB program that incorporates an adaptive minmax wavelet method. ${ }^{[9]}$ Subsequently, all the Raman spectra were smoothed, and their intensities were normalized with respect to the peak of the highest intensity in the spectrum. To eliminate the influence of any artifacts that can occur during data processing, we excluded $100 \mathrm{~cm}^{-1}$ region of the spectrum at both ends, i.e. the region of the spectra used was from 600 to $1800 \mathrm{~cm}^{-1}$, which is commonly known as the 'biological window' in Raman spectroscopic studies.

To quantify the spectral differences among the various groups present in the data, we have analyzed the data using PCA and DFA, the commonly used chemometric analysis techniques in biomedical research. ${ }^{[10,11]}$ The PCA lowers the dimensionality of the original data and constructs new fewer variables that capture most of the variance in the original data. ${ }^{[10]}$ These new orthogonal variables basically represent the main independent trends in the original data set. In our PCA, 591 Raman intensity variables, separated by $\sim 2 \mathrm{~cm}^{-1}$ interval, from 600 to $1800 \mathrm{~cm}^{-1}$ were used as statistical variables, and the PCA reduced them to 18 principal components capturing $\sim 96.7 \%$ variance in the data set. The principal components were used as input variables for the DFA that expresses the data in terms of number of discriminant functions (DFs), which is one less the number of groups. ${ }^{[11]}$
To assess the utility of FFPP tissues for diagnostic purpose, the Raman data were collected from the FFPP tissues obtained from nine different patients (Table 1) and used as training/reference set to test the data obtained from three patients. For the training set, the classification predictions based on Raman spectroscopy measurements using the leave-one-out method are crossvalidated with the 'gold standard' results of pathology.

\section{Results and discussion}

\section{Comparison of frozen and the corresponding deparaffinized tissues}

Figure 1 shows the mean Raman spectra of frozen and deparaffinized tissue sections of normal adrenal gland along with the pathology image. Both the tissues produce good quality spectra with quite sharp and well-defined bands. Clearly, there are noticeable significant differences in the spectra. To highlight the spectral differences, we also show the difference spectrum in Fig. 1. A reduction in the intensity of the bands at 698,1155 , $1439,1517,1656$, and $1738 \mathrm{~cm}^{-1}$ is evident in the deparaffinized tissue compared with the frozen tissue, whereas the bands around 1003 and $1613 \mathrm{~cm}^{-1}$ show increase in intensity. The Raman bands $\sim 1155$ and $1517 \mathrm{~cm}^{-1}$ arise from C-C and C C stretching in carotenoids, which are present in high concentrations in different human organs including the adrenal gland. ${ }^{[12]}$ The Raman bands at 1656 and $1738 \mathrm{~cm}^{-1}$ can be assigned to $\mathrm{CO}$ and $\mathrm{C}-\mathrm{O}$ stretching in lipids. In the literature, the band $\sim 1656 \mathrm{~cm}^{-1}$ is often assigned to amide I band of proteins. ${ }^{[13]}$ However, it can also arise from lipids. Thus, the Raman band at $1650 \mathrm{~cm}^{-1}$ is often broad in tissues as it has $\mathrm{CO}$ stretching contributions from proteins, lipids, and other molecular groups whose wavenumbers are slightly different. The 698 and $1439 \mathrm{~cm}^{-1}\left(\mathrm{CH}_{2}\right.$ bend) bands are two of the strong bands observed in cholesterol, and it is generally assigned to 'lipids' in the literature, as lipid also has a strong band $\sim 1440 \mathrm{~cm}^{-1}$. Figure 1 also shows the Raman spectra of $\beta$-carotene, fat, and cholesterol. A comparison of the Raman spectra of frozen and the corresponding deparaffinized FFPP tissue sections of normal adrenal gland clearly indicates a complete loss of lipid, cholesterol, and $\beta$-carotene. In addition, some new Raman bands appear in the region of $1530-1630 \mathrm{~cm}^{-1}$ and a significant enhancement in the intensity of $1003 \mathrm{~cm}^{-1}$ peak. It has been shown in a previous study that formaldehyde interacts with proteins leading to the formation of methylene cross-linking. ${ }^{[14]}$ Such chemical reactions may lead to changes in the protein configuration and conformation. However, our 


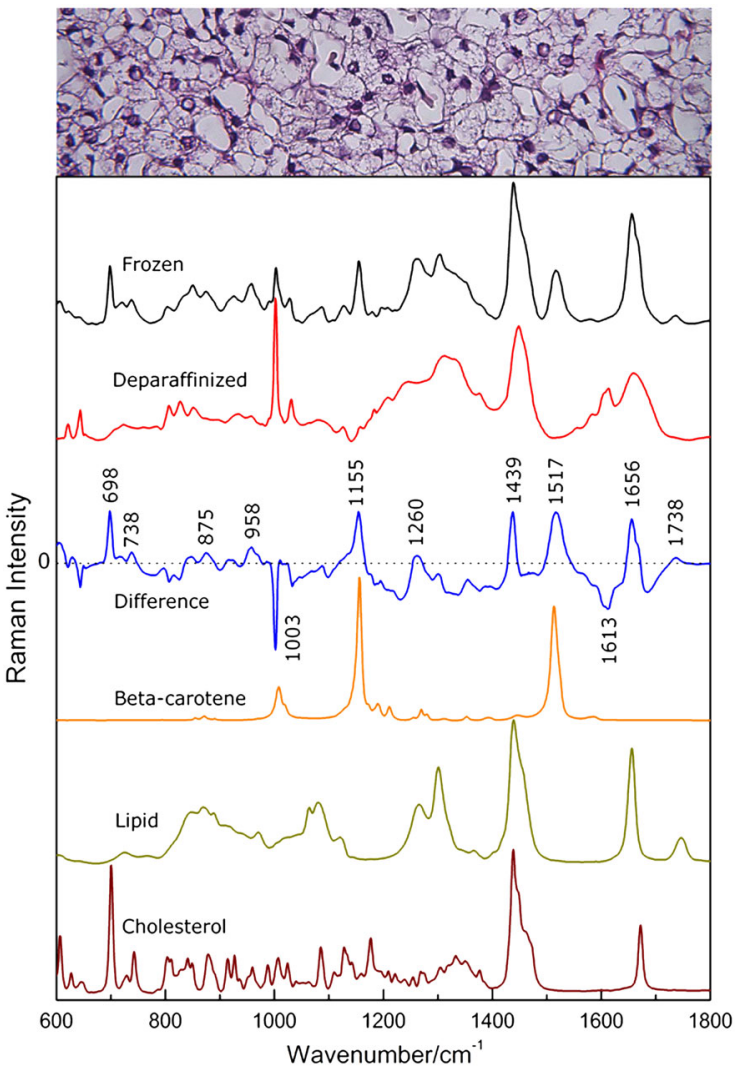

Figure 1. The mean Raman spectra of normal adrenal gland: from top to bottom, frozen, deparaffinized, difference, $\beta$-carotene, lipid, and cholesterol. Pathology image of the normal adrenal gland taken with $40 \times$ magnification.

observations show that the majority of the spectral features seem to be still preserved in the deparaffinized state with a reduction in the overall intensity of the spectrum.

Figure 2 shows the mean Raman spectra of frozen and deparaffinized tissue sections of neuroblastoma along with the

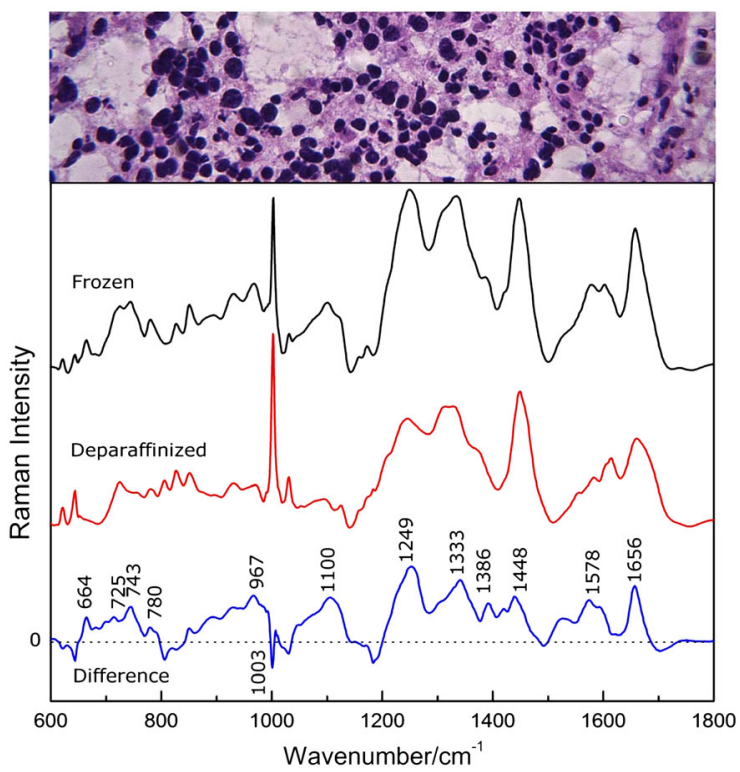

Figure 2. The mean Raman spectra of neuroblastoma: frozen, deparaffinized, and difference. Pathology image of neuroblastoma taken with $40 \times$ magnification. pathology image. The measurements were made specifically from the cellular areas avoiding stromal regions that are rich in collagen. Typical size of neuroblasts being around $10-20 \mu \mathrm{m}$ ( 2 to 3 red blood cell diameters ${ }^{[2]}$, the laser spot is confined to the area within the perimeter of the cell in most of the cases. A comparison of the Raman spectra of frozen and the corresponding deparaffinized FFPP tissue sections of neuroblastoma indicates that the majority of the spectral features are preserved in the deparaffinized state very similar to that of normal adrenal gland.

We have also compared the mean Raman spectra of frozen neuroblastoma (Fig. 2) with that of frozen normal adrenal gland (Fig. 1). Neuroblastoma lacks $\beta$-carotene, lipid, or cholesterol, and in addition, the Raman bands in the region of $\sim 1530-1630 \mathrm{~cm}^{-1}$ (nucleic acids), $1333 \mathrm{~cm}^{-1}\left[\mathrm{CH}_{3} / \mathrm{CH}_{2}\right.$ wagging (collagen, nucleic acids)], $\sim 1250 \mathrm{~cm}^{-1}$ (amide III/nucleic acids), $1100 \mathrm{~cm}^{-1}$ (C-N stretch, nucleic acids), 967, 780, 743, and $664 \mathrm{~cm}^{-1}$ (nucleic acids) are much enhanced in neuroblastoma compared with the normal adrenal gland. ${ }^{[15]}$ This indicates that the neuroblastoma cells are rich in nucleic acids and consistent with the fact that nuclear and cytoplasmic enlargement can occur during the progression of the tumor ${ }^{[16,17]}$ in neuroblastic cells.

Figure 3 shows the average Raman spectra of frozen and deparaffinized tissue sections of ganglioneroma along with the corresponding pathology image. The Raman data were collected from isolated ganglions (much larger than neuroblasts) while confining the laser spot within the perimeter of the cell. Hence, we expect the Raman bands in these spectra to originate from the nucleus and cytoplasm of ganglions. Several Raman spectra from deparaffinized ganglioneuroma tissue sections showed residual paraffin wax peaks, although the tissues were processed using the same procedures used in processing the normal adrenal and the neuroblastoma tissues. This is perhaps due to the intrinsic differences in the molecular interaction with paraffin wax in these tissues. It should be noted that the Raman spectra with residual wax peaks were not included in the analysis. A comparison of the Raman spectra of the frozen and the corresponding deparaffinized FFPP tissue sections indicates that

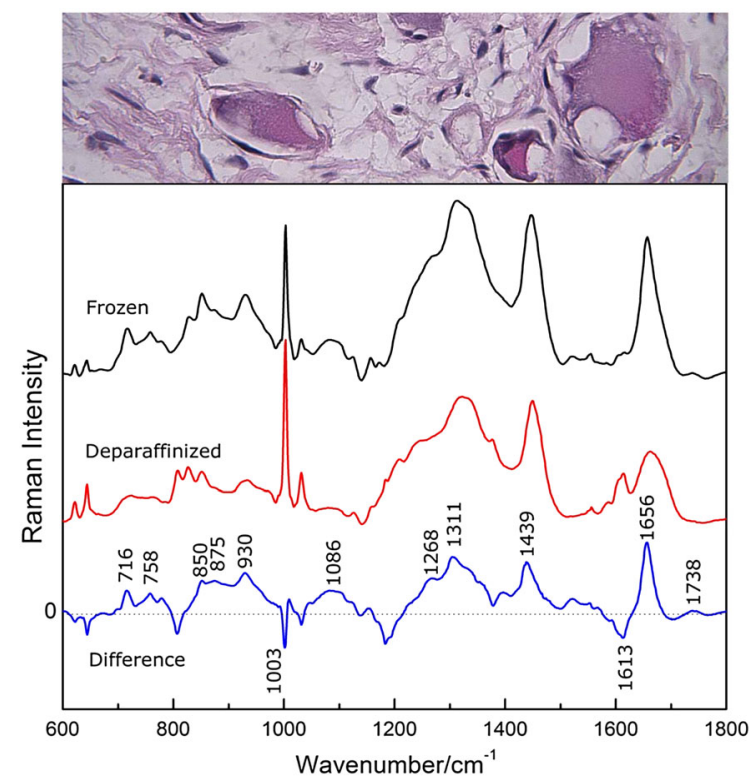

Figure 3. The mean Raman spectra of ganglioneuroma: frozen, deparaffinized, and difference. Pathology image of ganglioneuroma taken with $40 \times$ magnification. 
the majority of the spectral features are preserved in the deparaffinized state, very similar to neuroblastoma and normal adrenal gland.

We have also compared the mean Raman spectra of frozen ganglioneuroma (Fig. 3) with that of frozen neuroblastoma (Fig. 2) and found significant differences, especially in the spectral region of $1530-1630$ and $1200-1300 \mathrm{~cm}^{-1}$. In both of these spectral regions, the Raman bands in the spectra of neuroblastoma have higher intensity compared with the corresponding bands in ganglioneuroma. The bands in the $1530-1630 \mathrm{~cm}^{-1}$ region can be assigned to amino acid residues such as tryptophan and phenylalanine, and the bands in the $1200-1300 \mathrm{~cm}^{-1}$ region are assignable to amide III vibrations arising from the protein backbone. Thus, these intensity variations can be interpreted as an indication of the variation in the protein content in these tissues.

\section{Chemometric analysis}

To quantify the differences in the Raman spectra of normal adrenal, neuroblastoma, and ganglioneuroma tissues, we have analyzed the data using advanced chemometric methods such as PCA and DFA. Figure 4 shows the DF plot of the analysis using the Raman data for the frozen tissue sections. Clearly, the analysis separates the data into three distinct pathological groups representing neuroblastoma, ganglioneuroma, and normal adrenal with no overlap. As there are enough significant spectral differences in the spectra of the tissues as discussed earlier, it is not surprising to see this clear-cut separation. A DF plot of the analysis using the Raman data from the corresponding deparaffinized tissue sections also shows a similar grouping and separation. This is perhaps because each type of tissue in this part of the study came from a single patient and thus lacking any patient-topatient variability for a given pathology.

To address the variability of Raman spectral differences among patients of a specific pathology, we have studied normal adrenal, neuroblastoma, and ganglioneuroma archived tissues from nine additional patients (Table 1). The tissues were available only in the form of FFPP blocks without the corresponding frozen tissues. Figure 5 shows the average Raman spectra of tissues from each distinct pathology listed in Table 1 (Fig. S1 shows the same spectra along with their standard deviations). It is clear from Fig. 5 that the overall shape of the spectra is similar with observable

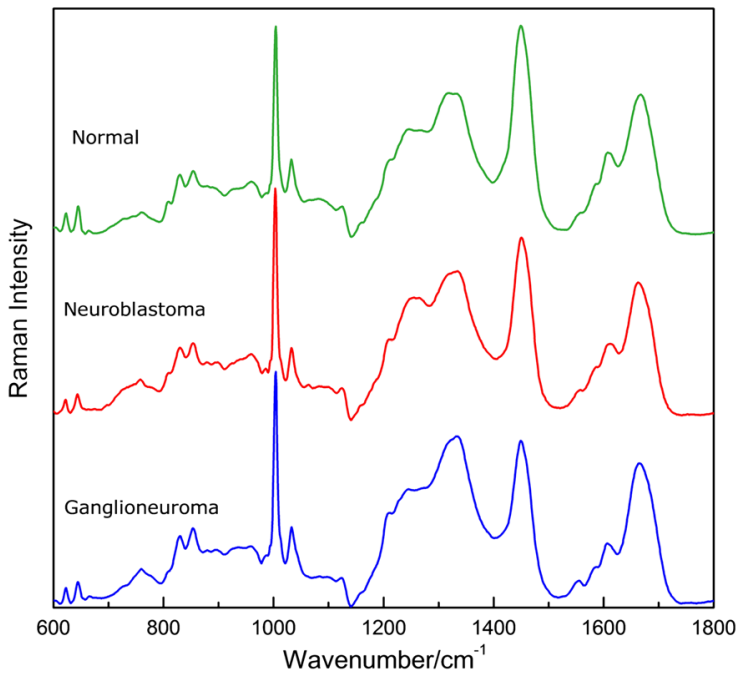

Figure 5. The mean Raman spectra of the archived formalin-fixed paraffin-processed tissues (Table 1). From top to bottom: normal adrenal gland, neuroblastoma, and ganglioneuroma.

differences in the intensity and broadening of the bands, which are quantified by the DF analysis. Figure 6 shows DF plot of the PCA-DFA analysis using the data shown in Fig. 5. The chemometric analysis does separate the spectra into three distinct groups belonging to each pathological state of the tissues, although the extent of spread around the group centroids is considerably larger compared with the analysis shown in Fig. 4 (b) for three patients. This could be attributed to the interpatient variability in the nine patients. The observed overlap between the ganglioneuroma and neuroblastoma can be understood in terms of the similarity of their spectra (Fig. 5) and the presence of some ganglions in neuroblastoma tissues. ${ }^{[2]}$ According to the leaveone-out classification method, the accuracies of the training set for normal adrenal, neuroblastoma, and ganglioneuroma are $100 \%, 89.7 \%$, and $95 \%$, respectively. We believe that addition of more data to the training set will improve the accuracy of identification. However, the observed overlap between the ganglioneuroma and neuroblastoma may continue to persist because of the presence of some ganglions in neuroblastoma tissues and their histologically similar appearance.
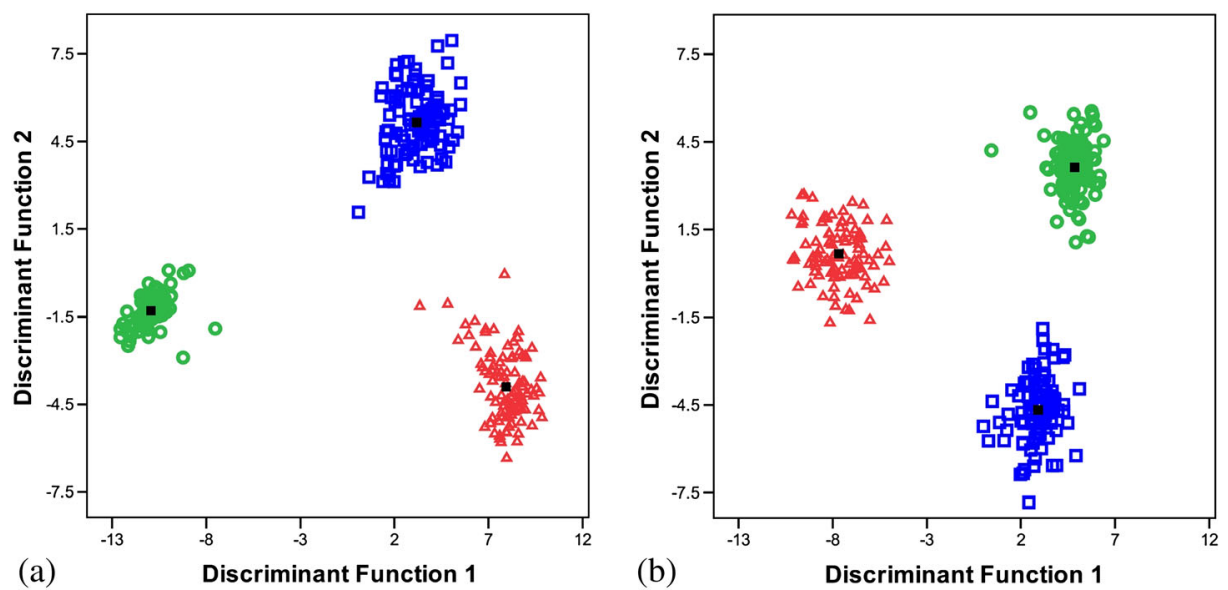

Figure 4. Discriminant function plots of (a) the frozen tissue data and (b) the deparaffinized tissue data: normal adrenal gland (circles), neuroblastoma (triangles), and ganglioneuroma (squares). 


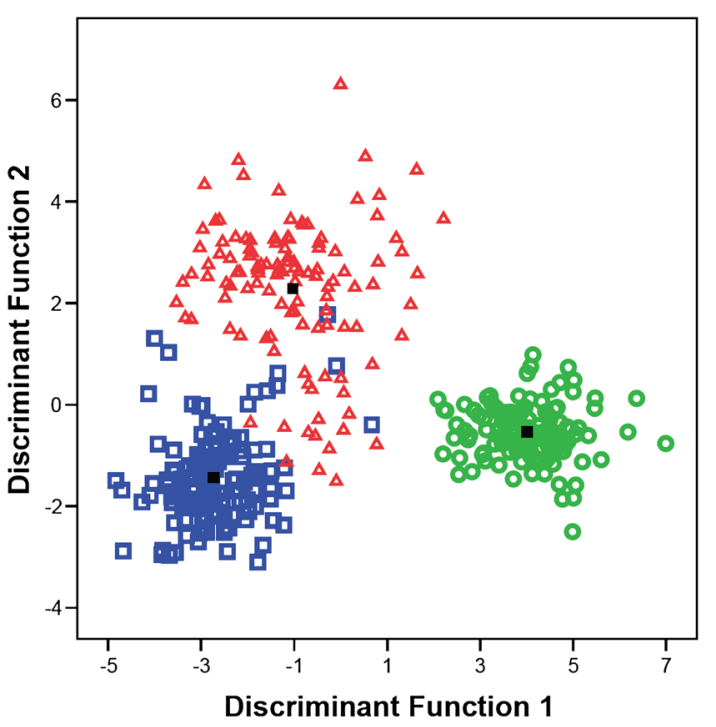

Figure 6. Discriminant plots of the archived formalin-fixed paraffinprocessed tissue data (Table I): normal adrenal gland (circles), neuroblastoma (triangles), and ganglioneuroma (squares).

Raman spectral data from the FFPP tissues from the nine patients (Fig. 6) were used as the training set in the chemometric analysis to validate the data from the deparaffinized tissues (the test set) obtained from the three patients in the first part of the study. The analysis grouped the normal adrenal test data correctly $>97 \%$ of the time with the data from the training set. A similar analysis of neuroblastoma test data correctly identified and grouped with the training set $100 \%$ of the time. However, the identification was very poor for the ganglioneuroma with $\sim 10.6 \%, 8.5 \%$, and $80.9 \%$ of the time identifying it as normal adrenal, ganglioneuroma, and neuroblastoma, respectively (Fig. S1). As seen in Fig. 6, the data from neuroblastoma has a much larger spread compared with the normal adrenal and ganglioneuroma due to the presence of some ganglions in neuroblastoma tissues and their histologically similar appearance, which may lead to poor categorization in the case of ganglioneuroma. It has been observed that chemotherapy treatment in neuroblastoma also induces ganglionic and stromal maturation and often neuroblastomas appear histologically similar to ganglioneuromas at the time of debulking surgery. ${ }^{[2]}$

\section{Conclusions}

We have investigated neuroblastoma and ganglioneuroma using Raman spectroscopy and compared their spectral characteristics with those of normal adrenal gland. In the first part of the study, the data were collected from the frozen and the FFPP tissues, obtained from the same tissue block, from three patients. The frozen tissue samples produce high-quality Raman spectra with distinguishable differences compared with the spectra from the corresponding FFPP tissues. The frozen sections of normal adrenal gland tissues show higher levels of carotenoids, lipids, and cholesterol compared with the neuroblastoma and ganglioneuroma tissues. A comparison of the Raman spectra of the frozen and deparaffinized tissues shows a significant alteration in biochemical of tissues, including the removal of carotenoids, lipids, and cholesterol in the adrenal tissues. The chemometric analysis of the data from both, the frozen and the deparaffinized, tissues show a clear-cut grouping. Our study shows that the deparaffinized tissues could be utilized for diagnostic research using Raman spectroscopy. However, its prediction accuracy is less than those of the frozen tissues due to the removal of some of the diagnostic biomarkers by formalin fixation and subsequent deparaffinizing processes. At present, it takes $\sim 4 \mathrm{~min}$ to record a full Raman spectrum ranging from 500 to $1900 \mathrm{~cm}^{-1}$. However, this time can be reduced by choosing a narrow wavenumber region relevant to the pathology of interest. Once the spectral database of relevant pathologies is established, the diagnosis of a new tissue can be performed in less than $5 \mathrm{~min}$.

In the second part of the study, a Raman spectral data set obtained from the deparaffinized tissues from nine additional patients was used as a training set against which the data from the deparaffinized tissues from the first part of the study, the test set, was validated using chemometric methods. The validation process successfully identified and correctly grouped the data with the training set in the normal adrenal ( $>97 \%$ of the time) and in the neuroblastoma ( $100 \%$ of the time) tissues, whereas the validation was not so strong for ganglioneuroma. This exploratory study indicates that the Raman spectroscopy combined with chemometric methods can be successfully used to distinguish neuroblastoma and ganglioneuroma in the frozen and deparaffinized tissues. The frozen tissues offer a better diagnostic outcome compared with the deparaffinized tissues because of their high-quality spectral features and no alteration and loss of biochemicals, which occurs in the process of formalin fixation, paraffin preservation, and subsequent deparaffinization of tissues for Raman studies.

\section{Acknowledgements}

We would like to acknowledge the kind support from the Richard J. Barber Foundation for Interdisciplinary Research. The authors also thank Dr M. D. Klein for providing access to their Raman facility at the Children's Hospital of Michigan and Dr Chung-ho Chang for reviewing the H\&E slides.

\section{Supporting information}

Supporting information may be found in the online version of this article.

\section{References}

[1] R.D. Riley, D. Heney, D.R. Jones, Clin. Cancer Res. 2004, 10, 4.

[2] N-K. V. Cheung, In Neuroblastoma, (Ed: S. L. Cohn), Springer, New York, 2005, 63-94.

[3] R. Rabah, R. Weber, G. K. Serhatkulu, A. Cao, H. Dai, A. Pandya, R. Naik, G. Auner, J. Poulik, M. Klein, J. Pediatric Surgery 2008, 43(1), 171.

[4] H. Wills, R. Kast, C. Stewart, R. Rabah, A. Pandya, J. Poulik, G. Auner, M. Klein, J. Pediatric Surgery 2009, 44, 386.

[5] H. Wills, R. Kast, C. Stewart, B. Sullivan, R. Rabah, J. Poulik, A. Pandya, G. Auner, M. Klein, J. Pediatric Surgery 2009, 44(6), 1152.

[6] Y-K. Min, S. Naito, H. Yamazaki, E. Kohda, H. Hamaguchi, In Raman Spectroscopy for Soft Matter Applications, (Ed: M. S. Amer), John Wiley and Sons, Inc., Hoboken, New Jersey, 2008, 279-281.

[7] E. O. Faolain, M.B. Hunter, J. M. Byrne, P. Kelehan, M. McNamara, H. J. Byme, F. M. Lyng, Vibrational Spectrosc. 2005, 38, 121.

[8] S. Devpura, J. S. Thakur, F. H. Sarkar, W. A. Sakr, V. M. Naik, R. Naik, Vibrational Spectrosc. 2010, 53, 227.

[9] A. Cao, A. K. Pandya, G. K. Serhatkulu, R. E. Weber, H. Dai, J. S. Thakur, V. M. Naik, R. Naik, J. Raman Spectrosc. 2007, 38, 1199.

[10] I. T. Jolliffe, Principal Components Analysis (Second edition), SpringerVerlag, New York, 2002. 
[11] W. R. Klecka, Discriminant Analysis, Series: Quantitative Applications in the Social Sciences, Sage Publication Inc., Thousand Oaks, CA, 1980.

[12] L. A. Kaplan, J. M. Lau,E. A. Stein, Clin. Physiol. Biochem. 1990, 8(1), 1.

[13] Z. Movasaghi, S. Rehman, I. U. Rehman, Applied Spectroscopy Reviews 2007, 42, 493

[14] J. A. Kiernan. Microscopy Today 2000, 00-1, 8.
[15] H. Deng, V. A. Bloomfeild, J. M. Benevides, G. J. Thomas, Biopolymers 1999, 50, 656.

[16] C. Kobayashi, H. Monforte-Munoz, R. B. Gerbing, D. O. Stram, K. K. Matthay, J. N. Lukens, R. C. Seeger, H. Shimada, Cancer 2006, 103 (1), 174.

[17] T. Tornoczky, D. Semuen, H. Shimada, I. M. Ambros, Pathology Oncology Res. 2007, 13(4), 270. 